\title{
TRACES HISTORIQUES DES ONZE MONASTÈRES FONDÉS PAR PACHÔME, PÈRE DU COENOBIUM, UN ESSAI DE SYNTHÈSE DES SOURCES VARIÉES
}

\section{Introduction}

Ayant établi sa première communauté vers 323, Pachôme n'a pas cessé de fonder les monastères régis sous son autorité et régularisés par les règles de la vie commune. Ainsi, la congrégation pachômienne consistait, vers la quatrième décennie du IV ${ }^{e}$ siècle, en neuf monastères d'hommes et deux couvents de femmes dispersés dans la vallée du Nil à distance de $175 \mathrm{~km}$ entre Panopolis et Latopolis de la Haute Egypte. ${ }^{1}$ Cet exploit méritait à Saint Pachôme le père du Coenobium et depuis la fin du $19^{\text {ème }}$ siècle, il y avait beaucoup d'études de son cénobitisme.

En effet, bien qu'on ait parlé beaucoup du cénobitisme égyptien inauguré au début du IV siècle, j'ai constaté des lacunes des études pachômiennes, dont la première concerne des traces que les onze monastères de Pachôme ont laissées dans l'histoire, en particulier après $V^{\mathrm{e}}$ siècle. Pour compléter cette lacune scientifique, j'ai essayé, dans cette étude, de faire une synthèse des données variées, c'est-à-dire littéraire, archéologique, voire papyrologique. Quant aux documents papyrologiques, je dois beaucoup à Monsieur Jean Gascou, Professeur à l'Université Marc Bloch (Strasbourg II) et Directuer de l'institut de Papyrologie. J'espère que cette étude de synthèse puisse contribuer à approfondir les études pachômiennes, surtout grâce aux informations papyrologiques.

(1) Cf. La première Vie grecque de Pachôme $=G^{1} 112$ 


\section{Les Monastères fondés par Pachôme}

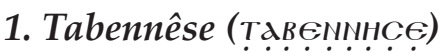

Après avoir passé sept ans de sa vie comme semi-anachorète sous la direction de son maitre spirituel Palamon, Pachôme s'installe vers 320 à Tabennêse, village abandonné à cette époque. Cette installation ni au désert ni en bordure de terre cultivée mais au sein d'un village annonce une des caractéristiques géographiques des monastères pachômiens qui seront situés principalement dans la plaine de la vallée du Nil. ${ }^{2}$ Le nom de Tabennêse était nouveau dans les milieux ascétiques de la deuxième décennie du $\mathrm{IV}^{\mathrm{e}}$ siècle. On entend dans la bouche de Théodore émanant d'un milieu semi-anachorétique l'expression 'Tabennêsiotes' pour se référer aux habitants ascétiques de Tabennêse. ${ }^{3}$ Les règles communautaires précisant la vie intérieure, ainsi que le mur d'enceinte qui est comme un cadre extérieur destiné à assurer la vie en communauté, devaient être à l'origine de ce fameux épithète, et plus tard le port dont ils disposaient contribuera beaucoup à la diffusion du terme. ${ }^{4}$

Pourtant ce dernier aspect ne caractérise pas l'ascèse communautaire des moines de Tabennêse du vivant de Pachôme. Il semble que les compilateurs ou les auteurs des Vies soient attentifs au mot 'bateau', quand la narration des récits leur obligent de l'écrire. Pachôme monte souvent sur une 'petite' barque pour se rendre à d'autres monastères. ${ }^{5}$ Deux bateaux essentiels pour la congrégation, rapportés par $G^{1} 113$, lesquels concernent la vente de nattes et l'achat d'autres besoins à Alexandrie, activités annuelles confirmées par Bo 107, étaient aussi qualifiés 'petit' dans la version bohaïrique (Bo 96). Par contre,

(2) Cf. J. Goenring, Withdrawing from the Desert : Pachomius and the Development of Village Monasticism in Upper Egypt, Harvard Theological Review 89 (1996) 267-286; IDEM, The Encroaching Desert: Literary Production and Ascetic Space in Early Christian Egypt, JECS 1.3 (1993) 281-296.

(3) La Vie bohaïrique = Bo 63 et $G^{1} 68$.

(4) Les Règles d'Ange (Histoire Lausiaque de Pallade 32, 2-7) reflète le nom des articles contraignants prescrit par Pachôme. Et la tradition copte et grecque ne manquent pas d'évoquer 'le mur de clôture' (Bo 42 et $G^{1} 42$ ). Tabennêse se situait 'sur le fleuve' (traduction d'une vingtaine Vies coptes de Pachôme: L. Th. Lefort, Les Vies Coptes de saint Pachôme et de ses premiers successeurs (Louvain, 1943) (Bibliothèques du Muséon, 16) 56).

(5) Bo 59; La cinquième Vie sahidique 53 et 54 (deux bateaux offerts à Pachôme sont aussi 'petits'). 
cette précision 'petit' a significativement disparu pour une barque qui entreprit, sous le gouvernement de Théodore, le voyage vers Alexandrie (Bo 183) et elle ne figure pas en général à l'époque des successeurs de Pachôme. ${ }^{6}$ Ce fait est très suggestif. Les compilateurs des Vies qui vivaient à la fin du $\mathrm{IV}^{\mathrm{e}}$ siècle veulent certainement mettre en relief un changement important par rapport aux débuts du cénobitisme : le fondateur ne disposait que de 'petites barques', tandis que les moines qui se vantent d'être ses fils spirituels ne l'ont pas suivi. Le premier monastère de Tabennêse, qui cède à Phbôou sa place vers 336/7, prévoit par sa propre localisation 'sur le fleuve' ses activités étendues sur le Nil, et dont Pachôme fit à son insu la préparation préliminaire.

\section{Le couvent féminin de Tabennêse ${ }^{7}$}

Après l'installation de la communauté à Tabennêse vers 323, Pachôme fonda bientôt une communauté féminine; sa sœur Marie est devenue la 'mère' ( $\mu$ í $\eta \varrho)^{8}$ des moniales qui vivent d'après les règles copiées par son frère Pachôme (MapIa, le nom n'est que dans la version bohaïrique 27). ${ }^{9}$ Le couvent de femmes était situé à peu de distance du village de Tabennêse. La date de sa création est différente selon les traditions : dans le texte copte, c'est avant 329, alors que $G^{1} 32$ (Atheniensis) la situe après 329-330, moment où Athanase d'Alexandrie visite Tabennêse. On s'incline à accepter la tradition grecque, comme on l'a vu plus haut.

Pachôme a confié à un certain Pétros le rôle d'intermédiaire entre les deux communautés de Tabennêse. Le responsable-intermédiaire des moniales meurt avant la grande pénitence de Théodore, et Titouiê,

(6) La mission de ce bateau était importante : «Il arriva encore qu'une fois une barque des frères, chargée d'étoupe destinée à leurs vêtements, sombra. Lorsqu'on apporta à notre père Théodore la nouvelle qu'elle avait sombré, les frères en furent fort tristes » (Bo 183).

(7) Voir aussi S. Elm, Virgins of God. The Making of Asceticism in Late Antiquity (Oxford, 1996) 289-296.

(8) La mère des moniales a, semble-t-il, une grande importance dans la vie des monastères pachômiens plus tardifs. Au cours du VI', un certain Jean, membre de la communauté d'Aphroditô, salue 'notre père commun très aimé

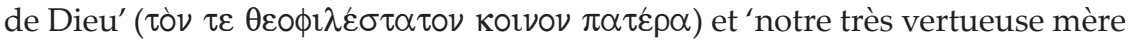

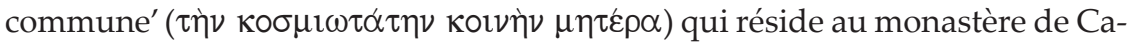
nope (P. Fouad 88, voir H. Marrou, 199).

(9) Cf. $G^{1} 32$ de l'Atheniensis. 
un des anciens, le remplace (Bo 27 ; voir aussi SBo 27, n. 6) ${ }^{10} \mathrm{Il}$ apparaît que ce dernier est décédé quelques temps après l'avènement de Théodore, et ensuite la visite des moniales est confiée à Apa Epônychos pour la communauté féminine de Tabennêse et celle de Phbôou.

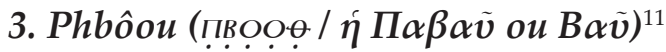

Vers 330, le surpeuplement de la communauté de Tabennêse conduit Pachôme à se diriger vers un autre village voisin abandonné appelé Phbôou pour y construire son deuxième monastère, également doté d'un mur d'enceinte ( $G^{1} 54$, Bo 49$) \cdot{ }^{12}$ Ce couvent de Phbôou qui dépendait de l'évéché de Diospolis Parva, se localisait à 2 mille $(3 \mathrm{~km})$ au nord-ouest de Tabennêse - le lieu est souvent mal localisé sur les cartes des monastères pachômiens - , et sa proximité permettait au fondateur résidant à Tabennêse de faire des visites fréquentes durant les premiers six années. En 336-337, c'est-à-dire, sept ans avant ce qu'on appelle sa 'grande pénitence' située en 344-346, Théodore fut établi à Tabennêse en tant qu'économe, et Pachôme séjournait à Phbôou qui devint ainsi à jamais la maison mère de toute la congrégation $\left(G^{1} 78\right.$, Bo 70).

Le monastère de Phbôou n'a pas disposé d'un port réservé à lui seul, puisqu'on n'en trouve pas traces, ni même d'un canal, et ce sur le plan littéraire comme archéologique. Il se trouvait dans une plaine, aujourd'hui fertile, et où la crue du Nil débordait souvent à l'époque de Pachôme. ${ }^{13}$ Cette localisation géographique explique indirectement

(10) $S B o=L a$ Vie de saint Pachôme, traduction de la vie bohaïrique (L. Th. Lefort (éd.), S. Pachomii vita bohairice scripta (Louvain, 1925) (CSCO, 89 ; Scriptores Coptici, 7) complétée par les Vie sahidiques par A. VeIlleux, La Vie de saint Pachôme selon la tradition copte (Bellefontaine, 1984) (Spritualité Orientale, 38).

(11) Voir aussi S. Tıмm, Fāw, dans: IDEm., Das christlichen-koptische Ägypten in arabischer Zeit (Wiesbaden, 1984) 947-957; R.-G Coquin et P. Grossmann, Pbow: History and Archaeology, dans: A. S. Atrya (éd), The Coptic Encyclopedia (New York-Oxford-Sydney, 1991) 1926-1929.

(12) Pour la chronologie, je me réfère à D. Chitty, A Note on the Chronology of the Pachomian Foundations, SP 2 (Berlin, 1957) (TU, 64) 381. Il ne faut pas penser que le nombre des moines pachômiens s'accroît rapidement; même après la fondation ou l'annexion de quelques monastères, les Vies nous renseignent qu'il y avait à peine 300 ou 360 moines dans la congregation (LEFORT, Les Vies Coptes de saint Pachôme et de ses premiers successeurs..., 180).

(13) L. Th. Lefort, Les premiers monastères pachômiens, exploration topographique, Le Muséon 52 (1939) 392-393. Le monastère de Phbôou est à 
son appui sur le port de Tabennêse pour la communication avec le monde extérieur, et plus souvent encore pour la visite des autres monastères filiales (cf. Bo 59 et 183, $S^{5} 53-54$ ). ${ }^{14}$ La tradition tardive décrivant la tête de la Koinonia pachômienne entre les mains de l'archimandrite soit de Phbôou soit de Tabennêse, s'explique sans aucun doute par le fait que le résidant de Phbôou doit toujours passer par le port de la communauté de Tabennêse pour naviguer sur le Nil.

Les pachômiens étaient fiers de leur 'monastère glorieux' de Phbôou, dès le début de son histoire, grâce à la construction de la grande basilique au milieu du Ve siècle. ${ }^{15}$ La basilique de Phbôou (l'actuel Fāw al-Qublī) a fait l'objet de fouilles menées par P. Grossmann. ${ }^{16}$ Et les explorations archéologiques ont distingué trois moments différents de construction de l'église : une ancienne, une deuxième à la fin du $\mathrm{IV}^{\mathrm{e}}$ siècle ou au début du $\mathrm{V}^{\mathrm{e}}$ siècle et une dernière après un demi siècle. Le premier oratoire de Phbôou à l'époque de Pachôme n'est connu que par les paralipomena ch. 32, et Bo 25 (cf. G ${ }^{1} 29$ ) concerne celui de Tabennêse. D'après Paralipomena 32, une fois construit l'oratoire, Pachôme pense qu'il est trop beau, et il ordonne aux frères de pencher les colonnes pour lui enlever de sa beauté. Le dernier édifice, consacré le 11 novembre 459 à l'époque de l'archimandrite Martyrios, était une basilique, comme toujours à cinq nefs, mais largement amplifiée par sa taille, $75 \mathrm{~m}$ de longueur et $37.5 \mathrm{~m}$ de largeur (elle peut accueillir au moins 2.000 personnes), avec beaucoup de colonnes de granit. L'agrandissement de la basilique de Phbôou parle, d'une manière indirecte, de l'économie fleurissante de la maison mère, à peu près au milieu du $\mathrm{V}^{\mathrm{e}}$ siècle. D'ailleurs, la dédicace de Timothée Elure a été l'occasion pour la littérature pachômienne d'assurer son attachement à la foi anti-

3 mille $(41 / 2 \mathrm{~km})$ du désert arabique du nord. La carte dans le Biblical Archeologist 1979, p. 201 localise, à ma connaissance, le plus exactement le monastère de Phbôou. Le canal du nord-ouest du village al-Fāw Qublī est moderne (CoQuin et Grossmann, Pbow..., 1928).

(14) $S^{5}=$ la cinquième Vie sahidique.

(15) A. Boud' Hors (texte copte et traduction) et R. Boutros (textes arabes et traductions), Timothée Elure. L'Homélie sur l'Eglise du Rocher attribuée à Timothée Elure, dans: PO 49, fasc. 1 (2001) 107 , 143, 145, 151-153.

(16) Coquin et Grossmann, Pbow, 1927-1929; P. Grossmann, The Basilica of Pachomius, Biblical Archeologist 42 (1979) 232-236. Pour connaître l'état de la question, voir J. Goenring, New Frontiers in Pachomian Studies, dans: B. A. Pearson et J. E. Goehring (éd.), The Roots of Egyptian Christianity (Philadelphia, 1986) 252-257. 
chalcédonienne comme on le voit aussis dans l' Allocution ${ }^{17}$ et l'Homélie attribuées à Timothée Elure.

Le stationnement des soldats de Justinien à la communauté de Phbôou, peu connu dans les milieux d'études pachômiennes, fait l'objet d'un grand intérêt. ${ }^{18}$ Ce numerus monté qui fut cantonné au cours du $\mathrm{VI}^{\mathrm{e}}$ siècle dans le centre monastique de Phbôou (B $\left.\alpha \tilde{v}\right)$ avait été composée, d'abord, d'un détachement de milice de Pharanites provenant d'une petite cité du Sinaï, Pharan, proche du monastère de Sainte Catherine, puis de soldats scythes. P. Lond. V 1735, 23-29, contient le contrat de vente d'une maison, et nous apprend quelques noms de membres de cette milice qui ont été des témoins de l'acte : Victor, Jacob et Théodore. ${ }^{19}$ Les soldats de Phbôou bénéficiaient de prestations fiscales prélevées sur les communautés de la Moyenne Egypte comme Aphroditô-Kom Ichaou (P. Flor III 297, 192, 219 et 302).

Parfois, les couvents pachômiens devaient verser les annones militaires pour la milice qui avait protégé entre autres leur maison mère contre les barbares. Le bureau des finances du village 'Aphroditô enregistre en 525-526 le versement des annones militaires du monastère pachômien de Tsmîne représenté par son moine, peut-être économe, Mousaios. ${ }^{20} \mathrm{~A}$ la liste des contributeurs s'ajoute le couvent pachômien de Stratonikis. Il est aussi notable que l'église épiscopale d'une Apollinopolis fut contributeur fiscal, au VI ${ }^{\mathrm{e}}$ ou au début du VII siècle, pour la milice de Phbôou, puisqu'on sait qu'elle paya 2 Solidi 21 c. pour la

(17) A. van Lantschoot, Allocution de Timothée d'Alexandrie prononcée à l'occasion de la dédicace de l'Eglise de Pachôme à Phbôou, Le Muséon 47 (1934) 13-56.

(18) Il est mis à jour par J. GAscou, à la fois corrigeant quelques lectures contradictoires proposées par R. RÉmondon et, d'autre part, publiant par la suite un nouveau témoin. Ainsi deux références: J. Gascou, P. Fouad 87: Les monastères pachômiens et l'Etat byzantin, BIFAO 76 (1976) 171-174 et IDEM., La Table budgétaire d'Antaeopolis (P. Freer 08.45 c-d), dans: J. LeforT et C. Morrison (éd.), Hommes et Richesses dans l'empire byzantin I (Paris, 1989) 309-310.

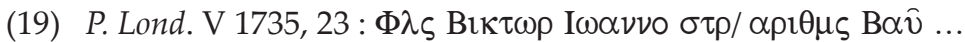

$24: \ldots \Phi \lambda / \operatorname{I} \alpha \kappa \omega \beta$ I $\omega \alpha \nu \nu \circ v \Phi \alpha \rho \alpha \rho \theta \theta \mu v \ldots$

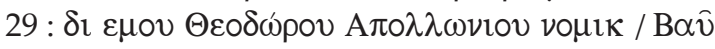

(20) P. Flor. 297 :

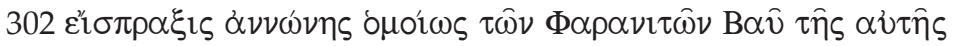

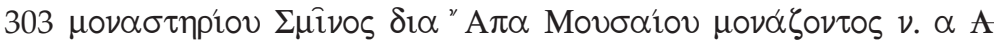
$\beta L^{\prime} \zeta v \gamma(\hat{\omega})$ 
prestation semestrielle d'une unité de scythes stationnée au couvent de Phbôou (P. Grenf II 95). ${ }^{21}$

Dans P. Freer $08.45 \mathrm{c}-\mathrm{d}$, la table budgétaire d'Antaeopolis, ville ducale, rédigée entre 533-543, nous permet de penser que le nombre de soldats établies à Phbôou ait été approximativement de cinquante ; car les annones sont quotidiennement de $50 .{ }^{22} \mathrm{Il}$ se pourrait que l'origine de ce stationnement des soldats dans le monastère de Phbôou du $\mathrm{VI}^{\mathrm{e}}$ siècle remonte au deuxième quart du Ve siècle. D'après l'Allocution attribuée à Timothée d'Alexandrie, Victor l'archimandrite qui est de retour de Constantinople à Phbôou en 431 est accompagné, entre autres, de 80 soldats que l'empereur Théodose II lui a offerts pour parer aux éventuelles attaques des barbares, et il se met avant tout à construire une citadelle autorisée d'avance par l'empereur au sein de sa communauté. ${ }^{23}$ Le Paralipomena 8-11 nous apprend d'autre part la présence des Blemmyes à la première moitié du IV siècle dans le désert proche du monastère de Phbôou. La vie de Šenoute $\S 89$ nous informe de son côté que pendant la première moitié du $\mathrm{V}^{\mathrm{e}}$ siècle, ils ont effectivement pillé quelques villages de la partie septentrionale du nome Panopolite; on sait aussi que l'armée locale d'Egypte byzantine avait surtout un rôle défensif contre les incursions barbares. ${ }^{24}$

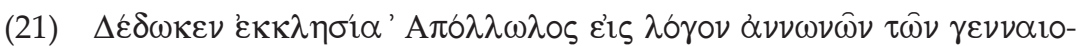

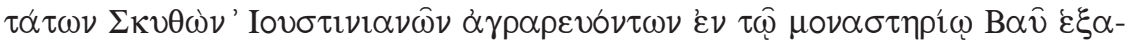

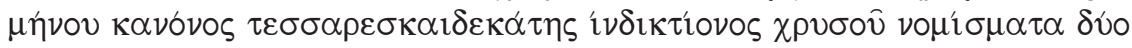

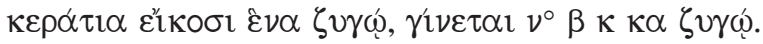

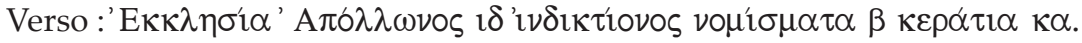

(22) P. Freer $08.45 \mathrm{c}-\mathrm{d}$,

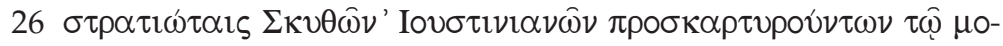

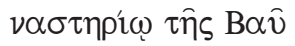

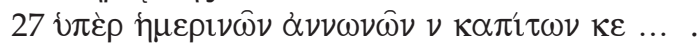

(26) «pour les soldats des Scythae Iustiniani en quartiers au monastère de Bau,

(27) pour 50 annones journalières et 25 capita... »

Une annone rémunère en principe un individu. Mais certains bénéficiaires, par exemple les officiers, touchaient de multiples annones, alors que d'autres, comme les valets de rémiment, se contentaient de demi-rations (J. GAsCOU, La Table budgétaire d'Antaeopolis (P. Freer $08.45 \mathrm{c}-\mathrm{d}$ ), dans: J. Lefort et C. MorRISON (éd.), Hommes et Richesses dans l'empire byzantin I (Paris, 1989) 290).

(23) van Lantschoot, Allocution de Timothée d'Alexandrie..., 43-45.

(24) Pour le rôle de l'armée byzantine d'Egypte, voir J. Gascou, L'armée, dans: C. Morrison (éd.), Le Monde byzantin I, L'Empire romain d'Orient 330-641 (Paris, 2004) 421-423. D’autre part, Les Blemmyes qui s'opposaient militai- 
Il se pourrait d'ailleurs qu'un nouveau village se formât autour de la communauté de Phbôou au moins avant le VIII" siècle. Dans un papyrus trilingue daté du VIII ${ }^{e}$ siècle et publié par A. Grohmann, on voit un certain Collouthos, fils de Phoibammon, signer en tant que chef du village de Phbôou. ${ }^{25}$ En même temps, un homme appelé Noah représente le village de Tabennêse, ce qui atteste également de la formation d'un nouveau bourg à Tabennêse abandonné au IV ${ }^{\mathrm{e}}$ siècle comme Phbôou. ${ }^{26}$ D'autre part, notre hypothèse est renforcée par quelques personnages connus dans le Synaxaire arabe. Les parents de Yousâb étaient anciens habitants de Fâw (Phbôou) avant de remonter le Nil jusqu'à Coptos. ${ }^{27}$ Fâw est aussi le village natal de Badâsyous. ${ }^{28}$ Ce dernier guérit la maladie d'une certaine Arsinoé, 'femme du premier magistrat de

rement aux Romains (par exemple, troisième guerre blemmye en Thébaïde entre 563-568), ne leur étaient pas toujours hostiles. Aux époques byzantine et arabe (ils sont connus souvent sous le nom de Beja à l'époque arabe (W. Y. Adams, Beja Tribes = Blemmyes, dans: The Coptic encyclopedia, 373-374), une partie de ce peuple était sédentarisée sur les marges de la vallée du Nil entretenant avec eux des relations souvent pacifiques (J. GAscou, Militaires étrangers en Egypte byzantine, BIFAO 75 (1975) 206); Pallade parle, au début du $V^{e}$ siècle, des Blemmyes établis près des moines de la communauté de Tsmîne (Histoire Lausiaque, ch. 32, 10). Pour la différence des recensions, voir l'édition de D. Butler, p. 210, n. 55). Cf. pour les couvents-forteresses du Moyen Age, voir M. H. Torp, Mûrs d'Enceinte des monastères coptes primitifs et couvents-forteresses, Mélanges d'Archéologie et d'Histoire 76 (1964) 173-200.

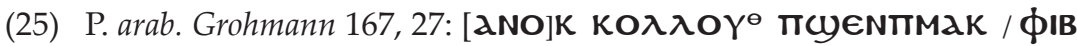
премтвоOY тістнхе †

(26) Il est sûr que Collouthos n'est pas l'archimandrite de Phbôou. Apa David est présente comme archimandrite du monastère d'Apa Zénobios (ama adYId mapMaN MTMON Nama ZHNOBIOC:167, 20), Philotheos

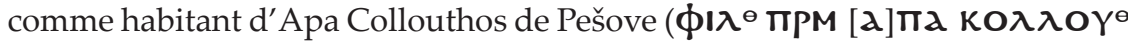
Mாє⿻yOүє : 167, 22), Phoibamon comme celui du monastère d'Apa Leontios

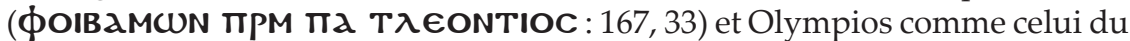

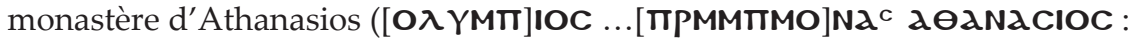
167, 35). Pourtant, s'agissant de Collouthos, fils de Phoibammon, habitant de Phbôou, il manque des indicessur son identité monastique.

P. arab. Grohmann 167, 19: [aNOK] NCUZE псунPE NтMak/ пєбш[C]]прMтавєNNHC€

(27) R. BAsset (éd.), Le synaxaire arabe Jacobite (rédaction copte), PO 3 (1909) 283.

(28) Idem, Le synaxaire arabe Jacobite (rédaction copte) III, PO 11 (1915) 666. 
Phabâou, sans doute dérivé du grec $\Pi \alpha \beta \alpha \tilde{v}{ }^{29}$ Enfin, le village existe encore aujourd'hui, tandis que le couvent de Phbôou attesté encore au VIII ${ }^{\mathrm{e}}$ siècle fut, dit-on, détruit par al-Hākim (996-1021).

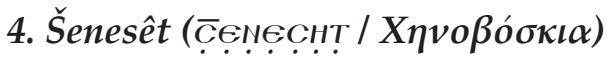

La communauté de Šenesêt ou Chénoboskia (l'actuel al-Qasr), qui existait probablement déjà vers 321 et se trouvait du même côté du Nil que Tabennêse-Phbôou est le premier couvent annexé, peu après 329, sous la direction de Pachôme. ${ }^{30}$ A l'origine, le père de la communauté de Šenesêt était un certain Ebonh ( $\mathbf{E B C N Z}$ : Bo 50) ou Epônychos

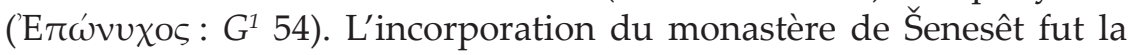
conséquence de la renommée des règles imposées par Pachôme aux monastères de Tabennêse-Phbôou. Pachôme lui-même dirigea, au début, les frères de Šenesêt en s'y rendant souvent (Bo 50), car le village était seulement à $12 \mathrm{~km}$ en aval de Phbôou. D'abord, il établi les chefs de maison et les seconds (Bo 50), et peut-être quelques temps après,

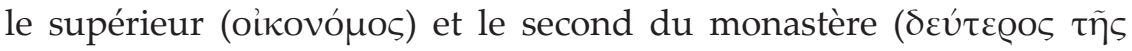

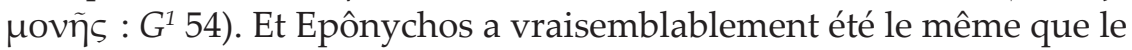
père des moniales sous le gouvernement de Théodore.

L'annexion de ce monastère constitue un tournant dans la vie de la Kononia pachômienne, parce qu'il ne s'agit plus d'un village désert

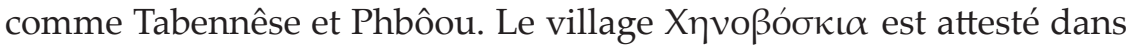
les papyrus à partir du II ${ }^{\mathrm{e}}$ siècle. Le $P$. Lond. II 196, qui rapporte un

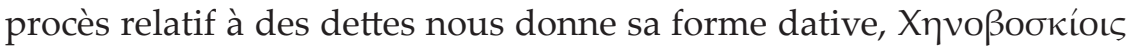

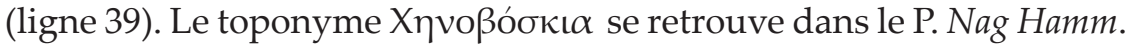
$(1,4$ et 31,3$)$ datée de la fin du III siècle ou du début du IV siècle, et

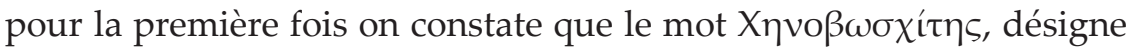

(29) Selon la traduction latine de I. Forget: Arsinoé est « principis nobilis ex incolis Fāu uxor » (I. Forget, Synaxarium Alexandrinum, I (Louvain, 1932) (CSCO, 78) 397).

(30) Lors d'une rencontre, peut-être en 387, d'Horsièse avec Théophile d'Alexandrie, le premier dit que soixante-six ans se sont écoulés depuis qu'il a pris l'habit monastique, ce qui signifie que son monastère d'origine qui est Šenesêt, doit exister en 321 (voir, la 21ème Vie Sahidique, Lefort, Les Vies Coptes de saint Pachôme et de ses premiers successeurs, 393). Pour toutes les variantes

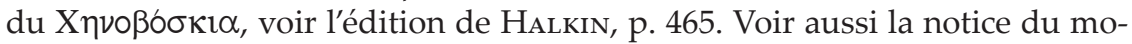
nastère de Chenoboskiôn dans H. GaUthIER, Nouvelles notes géographiques sur le nome Panopolite, BIFAO 4 (1905) 57-59. 
un 'habitant de Chénoboskia' $(153,9) .{ }^{31}$ Encore dans P. Erl. Diosp. qui remonte au premier quart du IV siècle (313-314), on trouve le toponyme $X \eta v o \beta(1,120$ et $167 ; 3,24)$ du nome Diospolite Parva, qui était sans doute une forme abrégée du village $X \eta$ voßóokı $\alpha .{ }^{32}$ Les gens de Chénoboskia effectuèrent, selon ce document, un payement à Aionios de 10 talents d'argent pour Theodosios $(1,166-167) .{ }^{33}$ Les données papyrologiques suggèrent enfin que la communauté de Šenesêt était en train de se développer évidemment en relation avec le village, non seulement avant l'incorporation dans la Koinonia pachômienne, mais aussi après son annexion qui se situe au début des années trente du $I^{\mathrm{e}}$ siècle. Elle fut aussi la communauté d'origine d'Horsièse $\left(G^{1} 114\right)$.

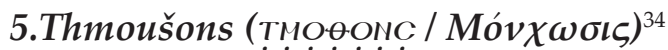

Il s'agit du deuxième monastère annexé à la Koinonia pachômienne (Bo et $S^{5} 51 ; G^{1} 54$ ). Au début, ce monastère fut organisé par Apa Jonas. ${ }^{35} \mathrm{Il}$ était aussi en 352 à l'origine de la révolte contre Horsièse, un des premiers successeurs de Pachôme ( $G^{1} 127-129$; S $\left.{ }^{5} 124-133\right)$. Au début du Ve siècle, Yūnās de Bahānis (Thmoušons) accompagne l'archimandrite Pachôme le Jeune pour aller à Alexandrie. ${ }^{36}$ Le $P$. Lond IV 1460, 54, registre d'impôts daté de 709 mentionne le village ( $\alpha$ Tò

(31) J. W. B. Barns, G. M. Browne et J. C. Schelton (éd.), NAG HAMMADI CODICES XVI, Greek and Coptic Papyri from the Cartonnage of the Covers (Leiden, 1981). A. VeIlleux mentionne aussi le village dans ce papyrus (A. Veilleux, Monasticism and Gnosis in Egypt, Semeia 58 (1992) 279).

(32) F. Miтthof, Ein spätantikes Wirtschaftsbuch aus Diospolis Parva, Der erlanger Papyruskodex und die Texte aus seinem Umfeld (P. Erl. Diosp.) (Leipzig, 2002) $4-5$.

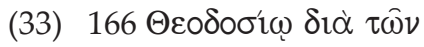

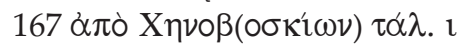

(34) Voir aussi S. Timm, Tmoušons, dans: Das christlichen-koptische Ägypten in arabischer Zeit, 2717-2720. Pour les variantes coptes et grecques du toponyme, voir les index de CSCO 99 et 100, p. 370 et de l'édition de HaLKIN, p. 462.

(35) Jonas est vivant à l'époque de Théodore ( $\left.G^{1} 123\right)$. Voir aussi, Lefort, Les Vies Coptes de saint Pachôme et de ses premiers successeurs, 116, n. 1.

(36) R. Coquin, Apa HĀMĀY Martyr pachômien au Ve siècle, Mélanges Serge Sauneron II Egypte post-paraonique (Caire : Institut français d'archéologie orientale du Caire, 1979) 155. 
Moov $\chi^{\circ} \pi \alpha \varrho^{\chi} \theta$ เvo $) .{ }^{37}$ Encore existant aujourd'hui, ce village est situé sur la rive occidentale du Nil à $7-8$ km au nord de Faršout.

\section{* Pmampesterposen}

Lefort veut le compter parmi les monastères fondés par Pachôme, ${ }^{38}$ ce auquel trois autres critiques modernes contestent. ${ }^{39}$

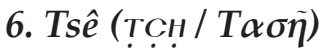

Chitty et Coquin comptent trois monastères d'hommes sur le territoire de Panopolis, alors que Lefort et Timm seulement deux. ${ }^{40}$ Je pense comme les premiers, étant donné que la tradition copte distingue clairement trois monastères dans le nome Panopolite, dont le premier est la communauté de Tsê.

Selon $S^{5}$ 52, une vision extraordinaire emmena Pachôme à fonder un couvent dans 'la région de Panopolis' (TKaZCyMIN), qui s'appelait Tsê (TCH- selon $G^{1}$ 83, T $\left.\alpha \sigma \tilde{\eta}\right)$. Le nom arabe Schedsina $(A m 569,2)^{41}$ de ce village copte Tsê n’a pas survécu. ${ }^{42} \mathrm{H}$. Gauthier écrit qu'Amélineau n'était pas sûr de l'existence de ce monastère. ${ }^{43}$ Mais le village Tsê

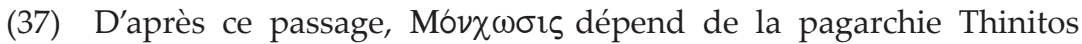
( $\pi \alpha \gamma \alpha \rho \chi i \alpha \theta$ ivíiov).

(38) Lefort, Les Vies Coptes de saint Pachôme et de ses premiers successeurs, 247, n. 3 .

(39) «Il est donc fort possible, ce n'est certes qu'une hypothèse, que Pma mpesterposen n'ait été qu'une dépendance, où résidaient seulement quelques moines, du grand couvent de Šensêt / Chénoboskia » (R. Coquin, Un complément aux Vies sahidiques de Pachôme: Le Manuscrit IFAO copte 3, BIFAO 79 (1979) 237); Chitty, A Note on the Chronology..., 383; Veilleux, La Vie de saint Pachôme..., 271, n. 4.

(40) Chitty, A Note on the Chronology of the Pachomian Foundations..., 382-383; Coquin, Un complément aux Vies sahidiques de Pachôme..., 233-237; Lefort, Les Vies Coptes de saint Pachôme et de ses premiers successeurs..., 247, n. 3 et 379-407; Tıмм, dans: Das christlichen-koptische Ägypten in arabischer Zeit..., 655.

(41) Am = E. Amélineau (édition et traduction), Monuments pour servir à l'histoire de l'Egypte chrétienne au IVe siècle. Histoire de saint Pakhôme et de ses communautés. Documents coptes et arabe inédits (Paris, 1889) (Annales du Musée Guimet, 179).

(42) E. Amélineau, La géographie de l'Egypte à l'époque copte (Paris, 1893) 485-486. D’après Amélineau, le mot Schedsinâ est composé du premier mot 凹€ T et d'un deuxième inconnu (ibid., 428-429).

(43) H. Gauthier, Nouvelles notes géographiques sur le nome Panopolite, BIFAO 4 (1905) 94. 
dans le nome Panopolite - il y avait plusieurs villages qui portaient le même nom - est attesté dans un papyrus du début de l'époque arabe

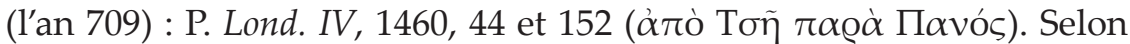
Timm, ce monastère pachômien de Tsê devait se situer vraisemblablement aux environs de l'actuel aş-Şawãmi'a Šarq, approximativement à $8 \mathrm{~km}$ au nord de Akmîm, où il existe aujourd'hui une église sur des ruines, vraisemblablement de ce monastère. ${ }^{44}$

\section{Panopolis (Šmin / Пavós)}

D'après la tradition copte ( $S^{5} 54$ et IFAO copte 3, 54, également $A m$ 569-570), Arios, évêque orthodoxe de Šmin (CyMIN)-Panopolis demanda à Pachôme de fonder un couvent dans sa ville. Selon la tradition copte, les gens envieux attaquèrent le mur d'enceinte pour le détruire, mais un ange l'entoura comme pour en faire un mur de feu, ce que la tradition grecque n'accentue pas. Pourtant, on cherchera en vain une mention de l'endroit précis où le couvent fut construit dans le nome Panopolite. Les Vies affirme que l'évêque a offert à Pachôme un terrain de construction, vraisemblablement avec des matériaux, car c'est ce que signifie la phrase «l'évêque leur désigna l'emplacement ». Cependant, si on considère les attaques renouvellées contre le mûr d'enceinte du monastère, notamment la visite des philosophes $\left(B o, S^{5}\right.$ et IFAO copte 3, 54 b et 55 ), il semble qu'il se trouve, parmi les monastères pachômiens panopolitains, le plus proche de la ville de Panopolis, et constitue un centre culturel au IVe siècle à côté d'Alexandrie.

L'attention portée au chapitre 32 de l'Histoire Lausiaque de Pallade permet de localiser le monastère de Šmin-Panopolis. Je m'attacherais à suivre l'avis de A. Veilleux, selon lequel Pallade ne monta pas audessus de Šmin. ${ }^{45}$ En effet, notre intérêt, un peu différent de celui de A. Veilleux qui voulait montrer le caractère non-pachômien des chapitres 32-34, se focalise sur le fait que Pallade rapporte un témoignage au sujet des monastères panopolitains de la congrégation pachômienne. Dans le chapitre 32,8 , après avoir présenté d'une manière concise le monastère de Phbôou, et Aphtonios, le second du monastère, il passe à la description des monastères panopolitains.

(44) Tıмm, dans: Das christlichen-koptische Ägypten in arabischer Zeit..., 654 655 et 87-88; voir aussi "Tsê" (pp. 2851-2852).

(45) A. Veilleux, La liturgie dans le cénobitisme pachômien au quatrième siècle (Rome, 1968), 138-146 et IDEM, Monasticism and Gnosis in Egypt..., 277-278. 


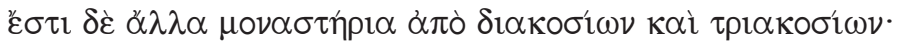

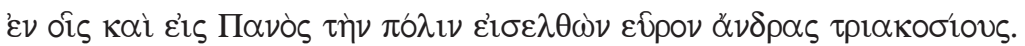
" Mais il existe d'autres monastères de deux cents et de trois cents.

Entre autres, étant entré à Panopolis, j'y trouvai trois cents hommes. »

(texte et traduction de Lucot, p. 221; texte identique à celui de l'édition de BARTELINK, p. 156)

Exilé en Egypte en 406, Pallade passa quelques années à Syène et à Antinooupolis avant de revenir en Galatie en $412-413 .{ }^{46}$ Quant aux monastères pachômiens, il ne visita que le monastère de Panopolis qui abritait trois cents d'hommes. ${ }^{47} \mathrm{Il}$ est difficile de savoir pourquoi il le choisit de préférence aux monastères de Kaior et Nouoi situés aux environs de Hermopolis Magna, sur la rive gauche du Nil, en face de la ville d'Antinooupolis. En tout cas, Pallade alla au couvent de Panopolis, sans doute en remontant le Nil de la ville d'Antinooupolis vers Panopolis. Les deux monastères de deux cents et de trois cents âmes pourraient rappeler les monastères de Tsê et de Tsmîne dont le nombre de moines est probablement transmis au pèlerin par la bouche des habitants du couvent de Panopolis. En effet, c'est 'aux confins

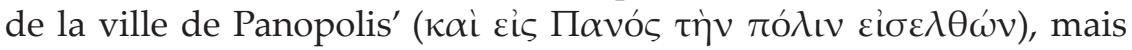
en dehors de cette ville, que Pallade trouva le monastère pachômien abritent trois cents hommes. On s'incline à penser que ce couvent est celui qui est fondé sur la sollicitation et grâce aux soutiens matériaux de l'évêque Arios.

Pourtant il faut se demander si la halte de Pallade au monastère de Šmin est crédible. Un peu avant le rapport de la visite de la ville de Panopolis, il introduit un certain Aphtonios. Son rapport sur ce personnage suscite un intérêt spécial.

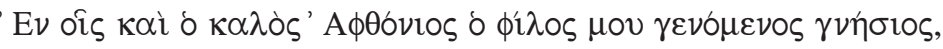

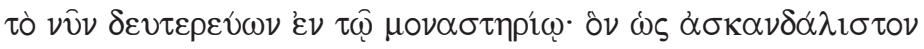

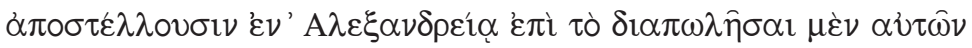
$\tau \propto े ~ \varepsilon ้ p \gamma \alpha$,

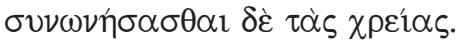

(46) A. Lucot, L'introduction de Histoire Lausiaque de Pallade, XIV; E. Amann, Pallade, dans: Dictionnaire de la Théologie catholique, t. 11-12, col. 1824.

(47) Il faut lire 300 au lieu de 3000 que Timm fournit dans sa notice d'Akhmim (Тімм, dans: Das christlichen-koptische Ägypten in arabischer Zeit..., 82). 
«Parmi eux, il y avait aussi le bel Aphtonios, qui est devenu mon ami intime

et qui pour le moment est le second dans le monastère. Comme il ne saurait être scandalisé,

ils l'envoient à Alexandrie pour vendre leurs ouvrages et acheter les choses nécessaires »

(texte et traduction de Lucot, p. 220-221; texte identique à celui de l'édition de BARTELINK, p. 156)

Pallade nous informe que Aphtonios a été envoyé à Alexandrie 'pour être consolé' à cause d'un conflit dont le caractère nous échappe'

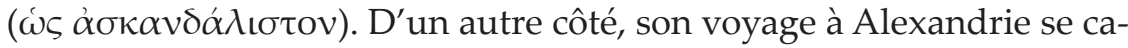
ractérise matériellement par la vente de produits manuels et l'achat des choses nécessaires. Ce témoignage a des parallèles dans les Vies. En 345, Zachée, chef des frères nautoniers demanda à Pachôme d'emmener Théodore en grande pénitence à Alexandrie, afin que ce dernier fut consolé (Bo 96). L'équivalent $G^{1} 113$ donne les deux motifs principaux du voyage à la capitale de l'Egypte : la vente des nattes et l'achat d'autres biens. Encore environ une cinquantaine d'années après, à l'époque du généralat de Bêsarion, apa Victor fut envoyé à Alexandrie après des troubles qui ont bouleversé la Koinonia, " pour qu'il reçut consolation $»{ }^{48}$ De manière significative, Aphtonios occupe donc une position considérable dans le monastère de Phbôou, comme l'étaient Théodore et Victor. Des exemples précédents rendent intelligible la cause de l'incident d'Aphtonios : un conflit autour de l'hégémonie dans la congrégation.

Cet Aphtonios fut l'ami de Pallade, et il serait donc la source orale de son rapport. Comment est-ce que Pallade n'ayant pas visité Phbôou arriva à connaître Aphtonios, moine de ce lieu ? La clé à ce problème doit être trouvée dans le rôle d'Aphtonios. Actuellement vũv), c'est-àdire certainement vers 406-411, Aphtonios est devenu le second du monastère de Phbôou. Au début du Ve siècle, il faut voir le rôle de l'économe général dans celui du sous-supérieur général de la communauté de Phbôou. Ce système est confirmée pour la première fois sous le gouvernement de Théodore. En 360, lors de la perquisition du duc Artémios à Phbôou en quête d'Athanase, le vice-supérieur de l'ordre hiérarchique après Théodore était un certain Psarphéin, nommé économe principal (oǐkovó $\mu$ о $\mu \varepsilon ́ \gamma \alpha \varsigma: G^{1} 138$ ), tandis que, selon l'ordre

(48) Lefort, Les Vies Coptes de saint Pachôme et de ses premiers successeurs..., 404. 
moral, Pékyssios, un des anciens de la première génération de la Koinonia, était, pour les frères de Phbôou, supérieur à l'économe principal. ${ }^{49}$

Cet indice permet de confirmer que, désormais, l'économe principal de la communauté de Phbôou est le second, dans la hiérarchie, du monastère ainsi que de la Koinonia pachômienne. Ce fait s'accorde bien avec notre hypothèse générale selon laquelle Théodore ne put être investi dans son généralat qu'avec son acceptation tacite des activités économiques des monastères, notamment commencées après la mort de Pachôme. ${ }^{50}$ Vers 352-360, les activités économiques des monastères pachômiens étaient tellement développées par rapport aux années précédentes qu'elles nécessitèrent qu'un économe général seconde l'archimandrite. Aphtonios, devenu le second du monastère de Phbôou, sous le gouvernement de Jonas le Doux ou de Pachôme le Petit, est ainsi devenu le grand économe. Et le rôle essentiel du grand économe de Phbôou consiste à préciser les travaux que doivent faire chaque économe des monastères. Dans l'exercice de cette fonction, les voyages vers les monastères de la Koinonia furent fréquents. C'est dans ce contexte qu'on peut situer des rencontres avec Pallade et Aphtonios. Les visites des monastères de Kahior et Nouoi, voisins de la ville d'Antinooupolis, sinon même des communautés panopolitaines, étaient autant d'occasions favorables pour lier amitié. En conclusion, je pense donc que le rapport de Pallade sur Aphtonios est authentique. De ce fait, selon le témoignage de Pallade, le monastère de Panopolis peut être localisé d'une manière approximative à la limite de la ville.

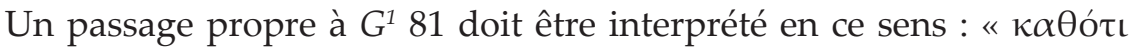

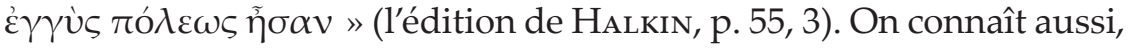
hors de la littérature pachômienne, quelques personnages qui ont vécu dans le monastère de Panopolis.

\section{Tsmîne (TCM!̣€ / Zuĩv)}

Après la fondation des communautés de Tsê et de Panopolis, le monastère de Thbêou appartenant à Pétronios fut annexé à l'ordre pachômien (Bo 56 et IFAO Copte 3, 56). Puis, Pachôme construisit un couvent

(49) Pékyssios est un des premiers huit disciples de Pachôme ( $\left.G^{1} 25-26\right)$. Par contre, Psarphéin succéda en 346 à Paphnuce, frère de Théodore, premier économe général $\left(G^{1} 124\right)$.

(50) Telle est l'hypothèse du chapitre II de notre étude. Cf. E. WirszyckA, Contribution à l'étude de l'économie de la congrégation pachômienne, Journal of Juristic Papyrology 26 (1996) 181. 
appelé Tsmîne (TCMINE) aux alentours de la ville de Panopolis $\left(S^{5} 56\right.$ et IFAO Copte 3, 57). Pachôme y établit très tôt Pétronios en lui confiant aussi le soin 'des deux autres monastères (N†KE CNOY †MMONH)' environnants, Tsê et Panopolis (Bo 56). ${ }^{51} \mathrm{Il}$ faut voir aussi une communauté de femmes sous la direction de Pétronios. Les deux autres Vies sahidiques mutilées transmettent également le nom du monastère, TCMINE ou TCMEINE dont Pétronios a été le supérieur jusqu'à la

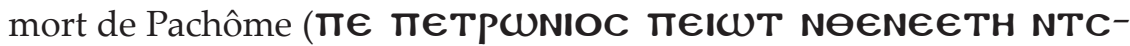
MINE, CSCO 99 et 100, p. 128, 19-20. TCMEINE dans la page 93, 9). La Vita Prima donnent ailleurs des variantes qui sont dûs à la difficulté

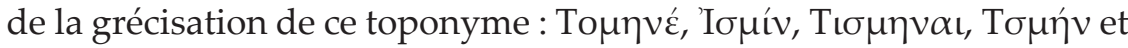
Touńv (voir l'index de l'édition de HaLkin, p. 465).

Malgré peu de renseignements dans les Vies, ce village, en particulier le monastère portant le même nom, laissa beaucoup de traces, notamment dans les papyrus, où, pour la première fois, il figure au III ${ }^{\mathrm{e}}$ siècle :

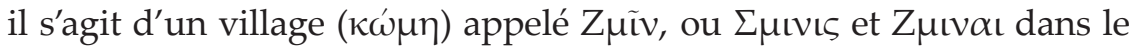
nome Panopolite (P. Froehner, Inv. 1127, A $2-3$ et 7-8, B 12). ${ }^{52}$ Le village

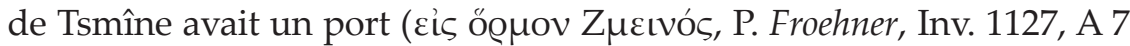
et B 12 ), et le P. Lond V 1686, 7-8 et le P. Cair. Masp. 67170, 5-8 situent le monastère de Tsmîne sur la rive occidentale du Nil en face de Panopo-

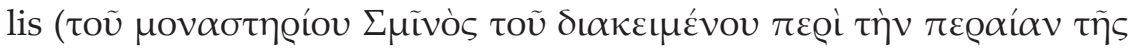

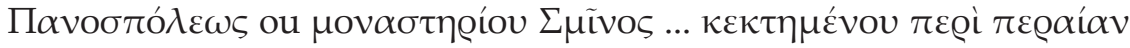

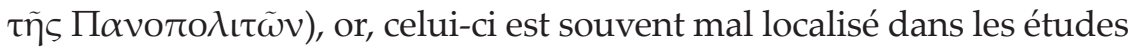
pachômiennes, notamment celles d'A. Villeux, Ph. Rousseau, H. Bacht

(51) IFAO copte 3 complète la lacune de la cinquième Vie sahidique, équivalent de Bo 56 : «€aq KCU $\boldsymbol{\lambda} \boldsymbol{\epsilon}$ ON Naq Mmpooycy N TKECNT€ N ZENE€TE ET ZHN EPOC : Il (Pachôme) lui confia aussi le soin des deux autres monastères, qui étaient proches de lui » (Coquin, Un complément aux Vies sahidiques de Pachôme..., 217, 14-18). Lefort a omis le mot 'autres' dans sa traduction de Bo 56 en étant convaincu qu'il n'y avait que deux monastères panopolitains (Снiтty, A Note on the Chronology..., 383; Coquin, Un complément aux Vies sahidiques de Pachôme..., 227, n. 7). Ainsi, l'agglomération des trois communautés panopolitaines se forma du vivant de Pachôme, comme une seconde Koinonia à cause de sa distance géographique qui était défavorable à des visites efficaces du fondateur.

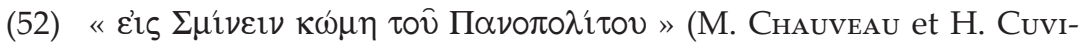
GHY, Les étiquettes de momies de la collection Froehner, Cahier de recherches de l'Institut de Papyrologie et d'Egyptologie de Lille 9 (Lille: Presses Universitaires de Lille, 1987) 71-72, n. 4). Le P. Lond. 604 B mentionne un habitant de Zmin,

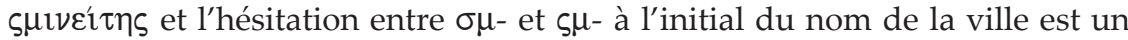
phénomène connu dans les papyrus grecs (Ibid., 72). 
et S. Elm..$^{53}$ D'autre part, au VIII e siècle, un certain Jean, chef de village de Tesmine (TECMINE) souscrit dans un document administratif trilingue où on se déclare sur l'innocence d'un collecteur d'impôts et de son équipe accusés d'oppression fiscale. ${ }^{54} \mathrm{~A}$ ma connaissance, c'est la première fois après les Vies de Pachôme que les toponymes coptes T€CMINE (Tsmîne) et CyMIN (Panopolis) apparaissent en même temps. ${ }^{55}$

A partir du VI ${ }^{\mathrm{e}}$ siècle, le toponyme Tsmîne est plus fréquent dans les documents en raison des activités économiques de son couvent pachômien. D'après le cadastre d'Aphroditô, document rédigé en 525-526, et publié par J. Gascou et L. MacCoul, la communauté de

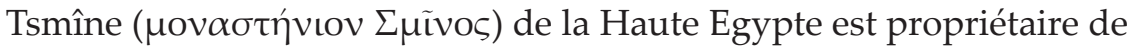
quelques terrains dans la région d'Aphroditô en Moyen Egypte, à un peu moins de $100 \mathrm{~km}$ en aval de Panopolis. ${ }^{56}$ Le monastère de Tsmîne y possède environ 33 aroures (égales à un peu plus de 8 hectares) de terre arable et de vignoble dont l'origine est variée et qui sont cultivés par deux responsables d'exploitation. Selon l'analyse de J. Gascou et L. Maccoull, les terres recensées représentent 5906 aroures (environ 1.500 hectares), et parmi les propriétaires sept autres monastères détiennent à peu près un tiers des surfaces cultivées. ${ }^{57}$ La propriété foncière du

(53) Ph. Rousseau, Pachomius. The Making of a Community in Fourth-Century Egypt (Berkely, 1985), 56; H. BACHT, Das Vermächtnis des Ursprungs, Studien zum frühen Mönchtum II (Köln, 1982) (Studien zur Theologie des geistlichen Lebens, 8) 295; Veilleux, La Vie de saint Pachôme, Carte des monastères pachômiens; ELm, Virgins of God..., 284.

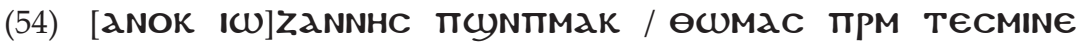
TICTY (P. Arab. Grohmann 167, 17). Ce papyrus n 167 est rédigé en original copte, récapitulé en grec, et traduit en arabe, mais mutilé.

(55) On voit 11 représentants de la ville de Panopolis (Šmin) se déclarer avec chaque nom suivi de ces deux formes: TPM Tाா 47, 48, 54, 61, 72, 73 et 74) ou Tracymin (167, 55 et 56).

(56) J. Gascou et L. MAcCoull, Le cadastre d'Aphroditô : P. Freer a-b, TM 10 (1987) 103-158. Nous allons citer les lignes ainsi que la traduction touchant le monastère de Tsmîne dont le nominatif est $\Sigma \mu \hat{\imath} \nu$.

(57) P. Freer

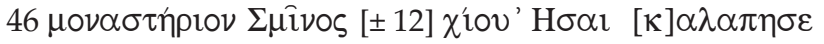

$$
\sigma \pi \circ(\rho .)(\alpha \rho .) \delta \eta^{\prime}
$$

(Monastère de Smin : 1/4 1/8 aroures de terre arable)

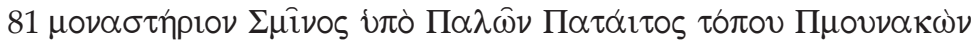

$$
\sigma \pi \circ(\rho .)(\alpha \rho .) L \delta \eta^{\prime} i \varsigma^{\prime} \dot{\alpha} \mu(\pi .)(\alpha \rho .) \alpha \mathrm{L}
$$

(Monastère de Smin aux mains de Palôs fils de Pataïs, lieu dit de Pmounakôn : 1/2 1/4 1/8 1/16 ar. de terre arable, 1 1/2 de vignes) 
monastère de Tsmîne n'est donc pas considérable dans ce document mais signifiante, puisqu'il s'agit de bien-fonds éloignés de l'emplacement de la communauté. Il y a également d'autres documents concernant le monastère de Tsmîne, dont la référence complète dans l'état actuel est fournit par l'éditeur du cadastre d'Aphroditô. ${ }^{58}$ Le $P$. Lond V 1690 est un contrat de location de terrain, signé le 29 août 527, en faveur d'Apollôs par Psaïos, le proestôs du monastère de $\Sigma \mu$ ĩv. Dans le $P$. Cair. Masp. 67170, daté de 564-565, deux fermiers, Aurelios Senouthès et Aurelois Ioulios, originaires du village de Tsmîne ( $\kappa \omega \dot{\mu} \eta \Sigma \mu \tilde{\imath} \nu$ s),

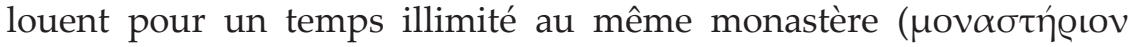
$\Sigma \mu \tilde{i v o s)}$ un jardin en vue de cultiver des légumes. Par ailleurs, le $P$. Lond V 1686 est l'enregistrement d'un contrat conclu, le 7 nov. 565, par lequel le monastère acquiert un droit de propriété sur trois aroures de terres appartenant à Dioscoros, notable d'Aphroditô, lesquels se trouvaient dans la partie méridionale du même village. Selon un registre d'impôts provenant du village d'Aphroditô, un fermier, responsa-

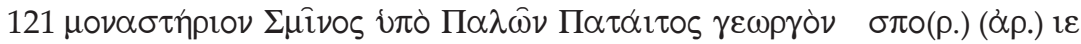
(Monastère de Smin aux mains de Palôs fils de Pataïs fermier : 15 ar. de terre arable)

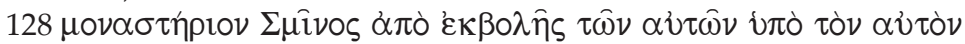
$\sigma \pi \mathrm{o}(\rho).(\alpha \dot{\alpha} \rho) \mathrm{L}$

(Monastère de Smin, par suite d'expulsion des mêmes - héritiers de Dios fils de Truphiodôre, L. 127-, aux mains du même - Palôs fils de Pataïs : 1/2 ar. de terre arable)

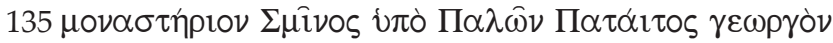
$\sigma \pi \mathrm{o}(\rho).(\dot{\alpha} \rho.) \propto \mathrm{L}$

(Monastère de Smin aux mains de Palôs fils de Pataïs fermier : 1 1/2 ar. de terre arable)

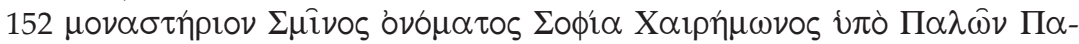

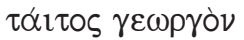

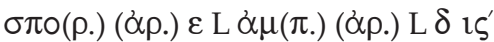

(Monastère de Smin, pour le compte de Sophia fille de Chairèmôn, aux mains de Palôs fils de Pataïs, fermier : 5 1/2 ar. de terre arable, 1/2 1/4 1/16 de vignes)

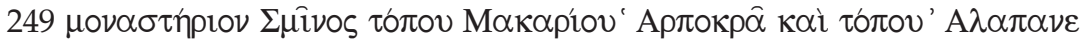

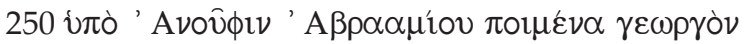

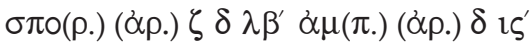

(Monastère de Smin, lieux-dits de Makarios fils d'Harpokras et d'Alapane, aux mains d'Anouphis fils d'Abraamios, berger et fermier, : 7 1/4 1/ 32 ar. de terre arable, 1/4 1/16 de vignes )

Gascou et MacCoull, Le cadastre d'Aphroditô..., 118. J. Gascou précise que les monastères sont absents des cadastres du IV siècle.

(58) Ibid., 137, note de la ligne 46. 
ble de l'exploitation d'une portion de terre paye l'impôt pour la communauté de Tsmîne (P. Flor. 297, 208). Ce dernier paye lui-même une fois, puis se fait représenter, toujours dans le même registre de taxes (P. Flor. 297, 294 et 376). Il faut mentionner également dans le même document que le monastère de Tsmîne versa les annones militaires au profit de la milice sinaïtique de cavalerie composée de Pharanites, qui stationna à la maison mère de Phbôou. ${ }^{59}$ On trouve pour finir des mentions, dans des documents de l'époque arabe, du tó ce qui désigne probablement un topos appartenant au monastère de Tsmîne. Le topos $\Sigma \mu$ ĩvos paye, en 706, par le biais de l'épouse du Kyrillos, l'impôt d'un demi Solidi et d'un demi artabe de blé (P. Lond. IV $1420,46)$ et probablement le même personnage dont le nom est cette fois-ci évoqué, Ezekiêl, paye un demi Solidi en 716 pour le même topos Touivos (P. Lond IV 1424, 14). ${ }^{60}$ Tous ces documents juridiques permettent de voir dans ce monastère un riche propriétaire de biens fonciers, en particulier dès le $\mathrm{VI}^{\mathrm{e}}$ siècle.

\section{La communauté féminine de Tsmîne (deuxième couvent des moniales)}

Dans son Histoire Lausiaque ch. 33, Pallade décrit une communauté féminine de Panopolis et raconte l'événement malheureux qui s'y passa. Timm, Lucot et Bartelink ont torts de supposer qu'il s'agit d'un monastère féminin de la région de Tabennêse-Phbôou. ${ }^{61}$ En effet, Ladeuze et $\mathrm{D}$. Butler veulent y voir une communauté de moniales de

(59) P. Flor. 297 :

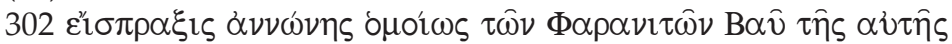

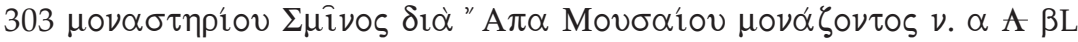
$\zeta v \gamma(\hat{\omega})$

Il faut lire $\mathrm{B} \alpha \bar{v}$ (le monastère de Phbôou) au lieu de la lettre mytérieuse $\beta$ (voir GAscou, P. Fouad 87..., 171-172).

(60) Le P. Lond IV 1420, 46 se lit : $\delta^{\tau} / \gamma \alpha \mu^{\varepsilon} \mathrm{K} v p ı \lambda \lambda \circ^{v} \int \mu^{\varepsilon} \tau 0^{\pi} Z \mu \imath v \circ \varsigma \nu^{\circ} \mathrm{S}$ $\nu^{\circ} \alpha \gamma l / \nu^{\circ} S \sigma l \alpha \rho^{\tau} S$

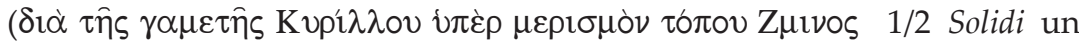
Solidi devient $1 / 2$ Solidi et $1 / 2$ artabe de blé).

Le $P$. Lond IV 1424, 14 est ainsi écrit : [ $\delta /] \gamma \alpha \mu^{\tau} \mathrm{Kvpı \lambda \lambda \circ \varsigma} \mathrm{I \varepsilon \zeta \varepsilon \kappa ı \eta \lambda}$

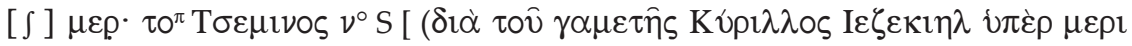

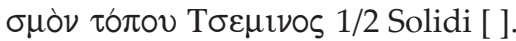

(61) Ттмм, dans: Das christlichen-koptische Ägypten in arabischer Zeit..., 2440; Lucot, Pallade, Histoire Lausique..., 33, 224 ; «nella regione di Tabennisi si trovavano tre monasteri femminili» (l'édition italienne de l'Histoire Lausiaque de Pallade, p. 362). 
Panopolis. ${ }^{62}$ Et il n'est pas difficile de saisir la cause de cette fondation, dans la mesure où la ville de Panopolis est, au IV et $\mathrm{V}^{\mathrm{e}}$ siècle, à côté d'Alexandrie, un des centres textiles et culturels. Ainsi il est raisonnable de penser que le récit légendaire de la rencontre entre un tailleur séculier et une moniale soit à attribuer à la communauté féminine de Panopolis, et non à celle de Tabennêse.

La tradition copte ne connaît pas cette communauté de femmes,

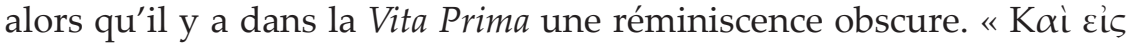

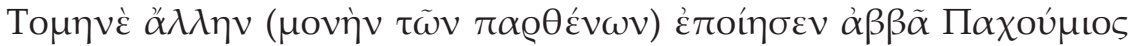
$\pi \varepsilon \varrho \iota \omega ́ v »\left(G^{1} 134\right.$; l'édition de HaLkin, p. 84, 27-28) : «et c'est à Tomêne que Abba Pachôme fonda une autre (communauté de femmes) dans son voyage». C'est la seule mention qu'on trouve au sujet de la nonnerie de Tsmîne dans toute la bibliothèque pachômienne, en principe réticente aux couvents de femmes et aux moniales. Le toponyme

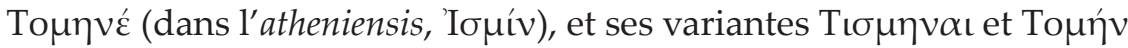
(l'édition de Halkin, pp. 56, 9 et 75, 9) n'est autre que le résultat de la grécisation du toponyme copte de Tsmîne. La localisation de Pal-

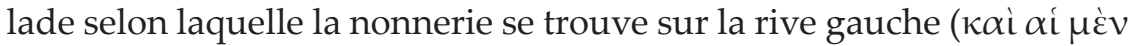

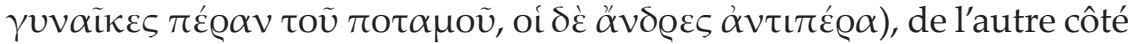
de Panopolis, est conforme aux données papyrologiques. Comme la première communauté féminine de Tabennêse, proche de Phbôou, la nonnerie de Tsmîne dont le catéchumènes étaient confiés aux didaskaloi

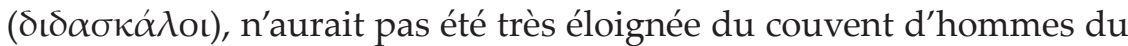
même village. ${ }^{63}$ En outre, on connaît, à travers une épigraphie, l'existence d'une moniale appelée Thekla originaire de la communauté féminine de Tsmîne.

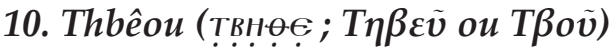

Après la fondation de la communauté de Panopolis, le monastère typiquement 'familial' de Pétronios fut incorporé dans la congrégation pachômienne ( $G^{1} 80$ et Bo 56). Selon Bo 56, la communauté de Thbêou se trouvait sur le domaine de ses parents. Et il est notable que, bien que Pétronios ait fait cadeau de tous ses biens à Pachôme, la terre ne figure pas dans la liste. D. Chitty pense que l'annexion de la communauté

(62) P. LAdeuze, Etude sur le cénobitisme pakhômien pendant le IV siècle et la première moitié du $V^{e}$ (Frankfurt 1961²) 177; D. Butlen, The Lausiac History of Palladius, VI 1-2 (Cambridge, 1904) (Texts and Studies, 6) pt. II, 211, n. 59.

(63) Pallade, Histoire Lausiaque 34, 4. Pour le terme didaskalos, voir ELm, Virgins of God..., 246-248. 
familiale de Thbêou n'est possible qu'après 339-340. ${ }^{64}$ Atteint par la peste, mais désigné comme successeur par Pachôme à ses derniers moments, Pétronios mourut le 19 ou 21 juillet 346 (Bo 130 et $G^{1} 117$ ) environs deux mois après la mort de Pachôme.

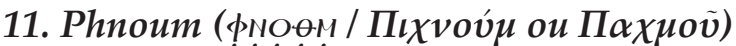

Il s'agit du dernier monastère fondé par Pachôme, qui se trouvait au sud de Latopolis (Bo et IFAO copte 358 et $G^{1} 83$ ). Il semble que la construction du mur d'enceinte du monastère provoqua, selon la tradition copte, la colère des chrétiens ainsi que celle de l'évêque de la ville. Lorsque Pachôme, convoqué entre 339 et 345 au synode de Latopolis à cause de son don de clairvoyance, fut victime d'une violence éclatante, il se réfugia dans ce monastère $\left(G^{1} 112\right) \cdot{ }^{65}$

\section{Conclusion}

En ce qui concerne Tabennêse, la première communauté de Pachôme, j'estime qu'il n'y a pas de grand chose. J'ai tenté de remarquer seulement la mention fréquente des 'petites barques' figurée dans les Vies, car les bateaux et les bateliers faisaient un rôle important dans l'histoire de la Koinonia pachômienne. Il faudrait une autre étude pour les activités maritimes sur le Nil des moines pachômiens. Le monastère de Phbôou qui a remplacé le rôle de Tabennêse en tant que maison mère, représente la congrégation pachômienne dans l'histoire, particulièrement avec la contruction de sa basilique du Ve siècle. Cette maison mère dont le nom se figure dans quelques documents papyrologiques du VI ${ }^{\mathrm{e}}$ siècle, est encore attestée même dans un papyrus trilingue daté de VIII ${ }^{\text {e }}$ siècle. Bau (Baṽ), autre toponyme grec de Phbôou est un des noms les plus fréquents dans les documents papyrologiques parmi les onze communautés de Pachôme.

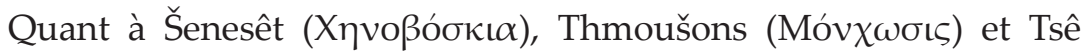
(T $\alpha \sigma \tilde{\eta})$, il n'y a pas de données siginicatives. J'ai essayé, pour la communauté de Panopolis, d'analyser, entre autres, quelques expressions enigmatiques dans l'Histoire Lausiaque de Pallade. On a arrivé à connaître le fait qu'il y avait souvent, dans la Koinonia pachômienne, un

(64) Chitty, A Note on the Chronology..., 382-384.

(65) A.-J. Festugière (introd. et trad.), La Première Vie grecque de saint Pachôme, dans: Les Moines d'Orient, t. IV/2 (Paris, 1965) 56-57; voir aussi АмéLINEAu (éd. et trad.), Monuments pour servir à l'histoire de l'Egypte chrétienne, 591-595 et 642-643. 


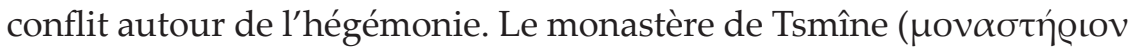
$\Sigma \mu \tilde{v}$ os) était un riche propriétaire de biens fonciers, surtout dès le VIe sicècle, comme on le voit dans quelques documents juridiques. Il est rarement mentionné dans les Vies mais les documents papyrologiques nous permettent de lui voir une grande importance économique parmi les monastères fondés par Pachôme. Pour les autres quatres monatères dont les deux sont féminins, leurs informations ne sont pas presques connues hors des Vitae pachomianae.

Les études pachômiennes restaient, durant le siècle dernier, principalement dans un domaine littéraire parfois complété par une fouille archéologique. Je crois que les données papyrologiques peuvent contribuer, avec d'autres sources traditionelles, à saisir mieux le développement des monastères pachômiens dans l'histoire et que la présente étude constitue un tel essai.

\section{Bibliographie}

\section{Vies de Pachôme}

Amélineau E. (édition et traduction), Monuments pour servir à l'histoire de l'Egypte chrétienne au IV siècle. Histoire de saint Pakhôme et de ses communautés. Documents coptes et arabe inédits (Paris, 1889) (Annales du Musée Guimet, 17).

Festugière A.-J. (introduction et traduction), La Première Vie grecque de saint Pachôme, dans: Les Moines d'Orient, t. IV/2 (Paris, 1965).

Halkin F. (édition des six Vies grecques), Sancti Pachomii Vitae Graecae (Bruxelles, 1932) (SH, 19).

Halkin F. (édition) et Festugière A.-J. (traduction), Le Corpus athénien de saint Pachôme (Genève, 1982).

Lefort L. -T. (traduction d'une vingtaine Vies coptes de Pachôme), Les Vies Coptes de saint Pachôme et de ses premiers successeurs (Louvain, 1943) (Bibliothèques du Muséon, 16).

Veilleux A. (traduction de la vie bohaïrique), La Vie de saint Pachôme selon la tradition copte (Bellefontaine, 1984) (Spritualité Orientale, 38).

\section{Sources papyrologiques}

P. arab. Grohmann Grohmann A. Arabic Papyri in the Egyptian Library, vol. 3: Administrative texts (Le Caire, 1938).

P. Cairo. Masp. Maspero J. Catalogue générale des antiquités égyptiennes du Musée du Caire (Milan, 1973; réimpression de l'édition de 1913).

P. Erl. Diosp. Miтtнof F. Ein spätantikes Wirtschaftsbuch aus Diospolis Parva, Der erlanger Papyruskodex und die Texte aus seinem Umfeld (P. Erl. Diosp.) (Leipzig, 2002). 
P. Flor. Vitelli G. Papiri Fiorentini, Documenti e Teste litterarii dell'Età Romanae Bizantina (Milan, 1915).

P. Fouad. Les Papyrus Fouad I, N¹-89, Publication de la société Fouad I de Papyrologie (Le Caire, 1939) (Textes et Documents, 3).

P. Freer $a-b$ GAscou J. et L. MACCoull, Le cadastre d'Aphroditô: P. Freer a-b, TM 10 (1987) 103-158.

P. Freer $c-d$ GAscou J. La Table budgétaire d'Antaeopolis (P. Freer 08.45 c-d). dans: J. Lefort et C. Morrison (éd.), Hommes et Richesses dans l'empire byzantin I (Paris, 1989) 279-313.

P. Froehner, Inv. 1127 A-B Chauveau M. et H. Cuvighy, Les étiquettes de momies de la collection Froehner, Cahier de recherches de l'Institut de Papyrologie et d'Egyptologie de Lille 9 (Lille: Presses Universitaires de Lille, 1987) 71.

P. Grenf II Grenfell P. et A. S. Hunt (éd.), New Classical Fragments and Other Greek and Latin Papyri (London, 1897).

P. Lond II Kenyon F. G. (éd), Greek Papyri in the British Museum, vol. 2 (London, 1898).

P. Lond IV Bell H. I. (éd.), Greek Papyri in the British Museum, vol. 4 (London, 1910).

P. Lond V BeLl H. I. (éd.), Greek Papyri in the British Museum, vol. 5 (London, 1917).

P. Nag Hamm. Barns J. W. B., Browne G. M. et J. C. Schelton (éd.), NAG HAMMADI CODICES XVI, Greek and Coptic Papyri from the Cartonnage of the Covers (Leiden, 1981) (Nag Hammadi Studies, 16).

\section{D'autres sources}

BASSET R. (éd.), Le synaxaire Arabe Jacobite (rédaction copte), dans: PO 3 (1909) 245-545; PO 11 (1915) 507-859; traduction latine: FORGET I. Synaxarium Alexandrinum, I-III (Louvain, 1932, 1926) (CSCO, 78; Scriptores Arabici 12; CSCO 90; Scriptores Arabici 13).

Boud'HORs A. (texte copte et traduction) et R. Boutros (textes arabes et traductions), Timothée Elure. L'Homélie sur l'Eglise du Rocher attribuée à Timothée Elure, dans: PO 49, fasc. 1 (2001).

LeIPoldt I. et E. W. Crum (éd.), Sinuthii archimandritae vita et opera omnia, I. Sinuthii vita bohairice (Louvain, 1906) (CSCO, 41. Scriptores Coptici, 1); Wiesmann H. Sinuthii vita bohairice (Louvain, 1951) (CSCO, 129; Scriptores Coptici 16).

Lucot A. (texte grec, introduction et traduction française), Pallade, Histoire Lausiaque (Paris, 1912); Butler D. C. The Lausiac History of Palladius, 2 vols. (Cambridge, 1904) (Contributions to Biblical and Patristic Literature, 6); l'édition italienne: Bartelink G. J. M. (texte et commentaire), 
M. BArchiesi (traduction), Palladivs Helenopolitanvs: Historia Lausiaca (Milan: Fondazione Lorenzo Valla, 1974).

\section{Etudes}

Adams W. Y. Beja Tribes = Blemmyes, dans: A. S. Atrya (éd.), The Coptic Encyclopedia (New York-Oxford-Sydney, 1991) 373-374.

Amann E. Pallade, dans: Dictionnaire de la Théologie catholique, t. 11-12, col. 1824.

Amélineau E. La géographie de l'Egypte à l'époque copte (Paris, 1893).

Васнт H. Das Vermächtnis des Ursprungs, Studien zum frühen Mönchtum II (Köln, 1982) (Studien zur Theologie des geistlichen Lebens 8).

Chauveau M. et H. Cuvighy, Les étiquettes de momies de la collection Froehner, Cahier de recherches de l'Institut de Papyrologie et d'Egyptologie de Lille 9 (Lille, Presses Universitaires de Lille, 1987) 71-80.

Chitty D. A Note on the Chronology of the Pachomian Foundations, Studia Patristica 2 (Berlin, 1957) (TU, 64) 379-385.

Coquin R. et P. Grossmann, Pbow: History and Archaeology, dans: A. S. Atrya (éd.), The Coptic Encyclopedia (New York-Oxford-Sydney, 1991) 1926-1929.

IDEm. Apa HĀMĀY Martyr pachômien au Ve siècle, dans: Mélanges Serge Sauneron II Egypte post-paraonique (Le Caire: Institut français d'archéologie orientale du Caire, 1979) 145-163.

IDEM. Un complément aux Vies sahidiques de Pachôme : Le Manuscrit IFAO copte 3, BIFAO 79 (1979) 210-248.

Elm S. Virgins of God. The Making of Asceticism in Late Antiquity (Oxford, 1996).

GAscou J. P. Fouad 87: Les monastères pachômiens et l'Etat byzantin", BIFAO 76 (1976) 171-174.

IDEm. La Table budgétaire d'Antaeopolis (P. Freer $08.45 \mathrm{c}-\mathrm{d}$ ), dans: J. Lefort et C. Morrison (éd.), Hommes et Richesses dans l'empire byzantin I (Paris, 1989) 279-313.

Idem. L'armée, dans: C. Morrison (éd.), Le Monde byzantin I, L'Empire romain d'Orient 330-641 (Paris, 2004) 421-423.

IDEM. Militaires étrangers en Egypte byzantine, BIFAO 75 (1975) 203206.

Gauthier H. Nouvelles notes géographiques sur le nome Panopolite, BIFAO 4 (1905) 39-101 et BIFAO 10 (1912) 89-130.

Goenring J. Withdrawing from the Desert: Pachomius and the Development of Village Monasticism in Upper Egypt, Harvard Theological Review 89 (1996) 267-286.

IDEm. The Encroaching Desert: Literary Production and Ascetic Space in Early Christian Egypt, JECS 1.3 (1993) 281-296. 
Idem. New Frontiers in Pachomian Studies, dans: Birger A. PeARson et James E. Goenring (éd.). The Roots of Egyptian Christianity (Philadelphia, 1986) 236-257.

Grossmann P. The Basilica of Pachomius, Biblical Archeologist 42 (1979) 232-236.

Ladeuze P., Etude sur le cénobitisme pakhômien pendant le IVe siècle et la première moitié $d u V^{e}$ (Louvain, 1898; Frankfurt 1961²).

Lefort L.-T. Les premiers monastères pachômiens, exploration topographique, Le Muséon 52 (1939) 392-393.

Rousseau, Ph. Pachomius, The Making of a Community in Fourth-Century Egypt (Berkely, 1985).

Tıмм S. Fāw, dans: Das christlichen-koptische Ägypten in arabischer Zeit (Wiesbaden, 1984) 947-957.

IDEM. Tmoušons, dans: Das christlichen-koptische Ägypten in arabischer Zeit (Wiesbaden, 1984) 2717-2720.

Inem. Tsê, dans: Das christlichen-koptische Ägypten in arabischer Zeit (Wiesbaden, 1984) 2851-2852.

Torp M. H. Mûrs d'Enceinte des monastères coptes primitifs et couvents-forteresses, Mélanges d'Archéologie et d'Histoire 76 (1964) 173-200.

van Lantschoot A. Allocution de Timothée d'Alexandrie prononcée à l'occasion de la dédicace de l'Eglise de Pachôme à Phbôou, Le Muséon 47 (1934) 13-56.

Veilleux A. Monasticism and Gnosis in Egypt, Semeia 58 (1992) 271306.

Wipszycka E. Contribution à l'étude de l'économie de la congrégation pachômienne, Journal of Juristic Papyrology 26 (1996) 167-210.

\section{SUMMARY}

Pachomius founded eleven monasteries, nine for man and two for woman during his life (290-346). In the Life of Pachomius, there are several informations about the cenobite communities scattered along the Nile. The Pachomian communities in the Life is limited chronologically only to the end of the fourth century. Nevertheless, other sources, in particular, the papyrological documents present the Pachomian Koinonia in the fifth and sixth century. In all, twenty-four local communities of the Pachomian Koinonia can be confirmed through the various sources of the 5th-6th century, and even 7th-8th century. This study focuses on the early eleven communities founded by Pachomius himself.

Concerning the first monastery of Tabennesis, the significant documents have not been found, and this phenomenon results mostly from 
the fact that Phboou, the second Pachomian monastery, played the role of the 'maison-mère'. The name of the Phboou is figured several times in the papyrological sources dated 6th and 8th century. Bau, Greek toponym of Phboou, is the most frequent name among the eleven monasteries in the papyrological sources. There are no important sources about Seneset (Chenoboskia), Thmousons (Monchosis) and Tse (Tase). On the other hand, several legal documents present that the monastery of Smin (Panopolis) was rich in the immovable property especially since the 6th century. Other pachomian monasteries were not almost known except Vitae Pachomii. The Pachomian study was focused generally on the literary sources, sometimes completed by archeological discovery. The papyrological materials can contribute to understand more the development of the Pachomian monasteries in the history. 